\title{
Bulbomembranous Urethra
}

National Cancer Institute

\section{Source}

National Cancer Institute. Bulbomembranous Urethra. NCI Thesaurus. Code C61124.

A portion of the urethra that extends between the apex of the prostate and the bulb of the corpus spongiosum and lies between the superior layers of the triangular ligament. 